\title{
Work stress, description of its main causes and consequences
}

\author{
Jorge Alex Pedraza Medina* \\ Clinical psychologist, Independent HR Consultant, Santa Cruz de la Sierra, Bolivia
}

\begin{abstract}
Currently the business world is constantly subjected to a competitive race in the labor market. The companies, in an effort to survive this competition, every day, raise higher quality standards, aligning its partners, the goal of achieving an optimum level of productivity and efficiency. This has promoted that in modern societies; the pace of modern life is fast and competitive, requiring the worker, to the high quantum of his life energy, enabling it to achieve both personal and work, goals and results. Derived from this context, in which to meet and deal with it, it aims to address this review article, the key features that allow understanding the distress (negative stress) and its devastating consequences; analyzing it as a serious risk factor for health, physical, intellectual and emotional, of any worker, regardless of race, age or gender.
\end{abstract}

\section{Summary}

Currently the business world is constantly subjected to a competitive race in the labor market. Companies, in an effort to survive this competition, each day, rise higher quality standards, aligning its partners, the goal of achieving an optimum level of productivity and efficiency. This has promoted that in modern societies, the pace of modern life, is accelerated and competitive, requiring the worker, to the high quantum of his life energy, enabling him to achieve both personal and work, goals and results. Derived from this context, in which to meet and confront, aims to address this review article, the key features that allow the understanding of distress (negative stress) and its devastating consequences; analyzing it as a serious risk factor for health, physical, intellectual and emotional, of any worker, regardless of race, age or gender.

\section{Stress, in the so-called global village}

Today, quality of life depends largely on the proper administration of that fuel element produced naturally in our body, called stress. When it is at the appropriate levels, it provides sufficient concentration and vital, vital energy for our daily activities and achieve our personal goals. In this global village, it is clear that the pace of modern life, fend for important scientific discoveries, technological advances and marketing money, among others, drags us towards pro to consumerist maelstrom, which promotes the desire for wealth to meet the real needs or fictitious of our life.

The coexistence of human beings on the planet, already well known by the sociologist Marshall McLuhan [1], as the "global village", provides a concept that blurs the boundaries of local and global coexistence. This becomes the progress of humanity through science, art and culture, in others. As a whole, this has allowed the events are local events and global knowledge and vice versa, being plausible to be known and socialized within seconds across the globe. The rise of computer technology has made it possible that humanity has taken the quantum leap over coming the barriers of geographical boundaries virtualizing human communication.
Making use of all this communicational swing is immersed human. Today any human being that lies in any parallel in the world, if you have the appropriate means of computer communication such as a computer or telephone device with access to the World Wide Web, you can quietly interact with anyone else in the globe hold similar circumstances.

\section{Technological evolution and human interaction}

The theories of the great natural scientist [2], through his observations and studies, spread important concepts such as evolution, adaptability and survival of the species. Darwin asserted that the fittest, strong or quick to adapt to changes in their environment efficiently so, was getting that preserve their species and progeny. Therefore, it seems that humanity in its desire to maintain its hegemony as the dominant species on the planet, increasingly strives to break more and more gaps separating their peers. Narrowing somehow, increasingly, that relationship as homogeneous human race despite their heterogeneous ontological characteristics as to species.

\section{Resilience and self-improvement}

Experts from the discipline of psychology in the twentieth century and early twenty-first, have emphasized in the innate capabilities to adapt to change, emotional strength and resilience to adversities that have arisen before mankind in many circumstances. Unfortunately, perhaps the most atrocious event and inevitably spacks more forcefully reality and life of people, forcing them to survive and adapt to change, whether the war.

In the Second World War, examples of the devastating humanitarian

Correspondence to: Jorge Alex Pedraza Medina, Clinical psychologist, Independent HR Consultant, Santa Cruz de la Sierra, Bolivia, Tel: +34 686399 661; Fax: +34 686399 661; E-mail: jorgeapedrazam@yahoo.com

Key words: syndrome, stress, distress, burnout, eustress.

Received: March 11, 2016; Accepted: April 23, 2016; Published: April 26, 2016 
crisis in terms of moral and civic values occurred. Psychologist that she experienced an important raw drama of the war, being captive and enslaved in a Nazi concentration camp during the Holocaust, fully exemplifies this line of thought. It is the professor in neurology and psychiatry, Victor Frankl [3], who after having observed his cellmates, subjected to forced labor, grotesque aberrations, dingy isolation, constant starvation, finally work in the middle of that total desolation, scorn and daily human distress, it concluded that only survived those in the midst of despair, had a meaning in their lives, which stay alive and fight, because in their heart of hearts they knew that survive that storm and reinvent their lives.

However it also noted, the antipode of the above concept, in which cellmates, lost all traces of hope and abandoned their struggle for survival thus easily, easily succumbing to his captor's enemies. This allowed him to build his speech therapy paradigm where specified that the human being only reaches happiness and fullness of his life, when it finds a real, genuine and meaningful sense for their existence.

\section{Paradigms, structures and superstructures}

Under a magnifying glass in retrospect, it is clear that every decade; humanity has glimpsed important paradigm shifts. Physical, humanist philosopher Mario Bunge, referring to such changes in science, as conceptual structures which in turn builds superstructures of ideas and theoretical constructs.

However, Bunge also asserted that from time to time within the scientific field, at the time of arise to new paradigm, some of the old structures can be overthrown in their theoretical foundations, losing force, and leading to the construction of a new paradigm that gives greater coverage and conceptual efficiency.

\section{Local economy in a global environment}

Under this perception human being comes to be incorporated as an agent of change within an increasingly globalized local economy, exchanging their physical and/or intellectual energy in exchange for adequate remuneration necessary to meet their personal needs and/or parent.

The vital energy that comes from human beings, bio psychosocial mixture of aptitudes and abilities, on the one hand and intelligence and motivation on the other, lead to the same, be a productive entity that contributes to its economy. The lure of subsistence, through its productivity, it promotes not only be versatile but above all the need to be efficient. In the current competitiveness was requires greater intellectual and emotional preparation to meet the challenges that organizations demand from their partners.

\section{Stress and its characteristics}

Outlining this perspective, the human being, as work organization, often sues certain demands from the corporate environment, which are among others, mainly the fact maintain its pace of work under a high level of productivity and efficiency, thus, achieve goals and economic and trade performance. Situation that under certain circumstances, can result in disease symptoms allusive to work stress and its consequences in various areas of their daily lives.

Therefore, it is logical to refer properly to burnout syndrome, transit is required, theoretically, the path denoting certain primary concepts and their parallel time, such as the stress, as well as specifically to the concept of job stress.
In the first instance, we decantaremos the generic concept of stress and its main features

To do this, we will name the physiologist, physician and researcher, Dr. Hans Selye [4] who through their biological research, based on observations of the early years of his medical training at the University of Prague, fed his Stress curious about.

These observations were revealing themselves, to maintain contact with the sick in the hospital of the University, in which mightily, I call attention to the fact finding certain similarities between the symptoms of their rats as specimens of diseased laboratory and expressing patients between their general symptoms, such as fever, headache, abdominal pain, weight loss, among others, morbid entity affecting them independently.

Therefore, I have decided to call this curious similarity observed, only sick syndrome as symptomatic. Circumstance that allowed him to establish a functional definition of stress, subsequently revealing it as "that state manifested by a specific syndrome, consisting of all nonspecific induced changes within a biological system."

Thus it was that in his later investigations revealed the concept that stress is nothing but the sum of all non-specific effects (i.e., on many organs, without selectivity) of factors (normal activity, producing agents of disease, drugs, etc.) that can act on an organism. These agents can cause stress, I called alarmogenos (stress factors), to differentiate the result which ended just denominating stress.

Also in his first presentations to the scientific community on such symptoms, I have said that it could be compared to the concept of resilience of an organism, under the pressure which becomes by external agents against its homeostasis.

Concept which alluded to the analogy observed in Newtonian laws of physics, revealing implications on the pressure and forces that interfere on the strength of a solid material and its ability to deform. And is that stress can deform the subject who suffers?, the answer is yes, figuratively.

We will make a brief reference to two sub-items from the stress, called Eustress positive which is a type of stress but, given the characteristics of coping and lead in resolving the situation. And the other, which is the one that interests us, disaggregate, called Distress or negative stress. The latter carries negative health consequences for its excessive physical, mental and emotional burden on any person who has it.

When the stressor agents overcome the resistance level of an individual, at the high pressure of a situation or sum of situations, if the person himself does not find routes resolution in the resistance phase, inevitably you can immerse yourself in a state of imbalance and lack of self-control physical and / or psychological, negating their ability to effective response to such a situation (Figure 1).

\section{Burnout syndrome, a variant Stress causes most damage to health}

Given the epigenesist and main characteristics of stress, we can continue to explain that workers may feel stress when the demands of your job are greater than their ability to cope efficiently and effectively. By not achieve the results required by their employment agency, it is common that the cluster on demand, entailing not only mental health but can also develop serious physical health problems such as musculoskeletal disorders or diseases of cardiovascular condition.

Here we can mention the work of the famous psychologist Herbert 


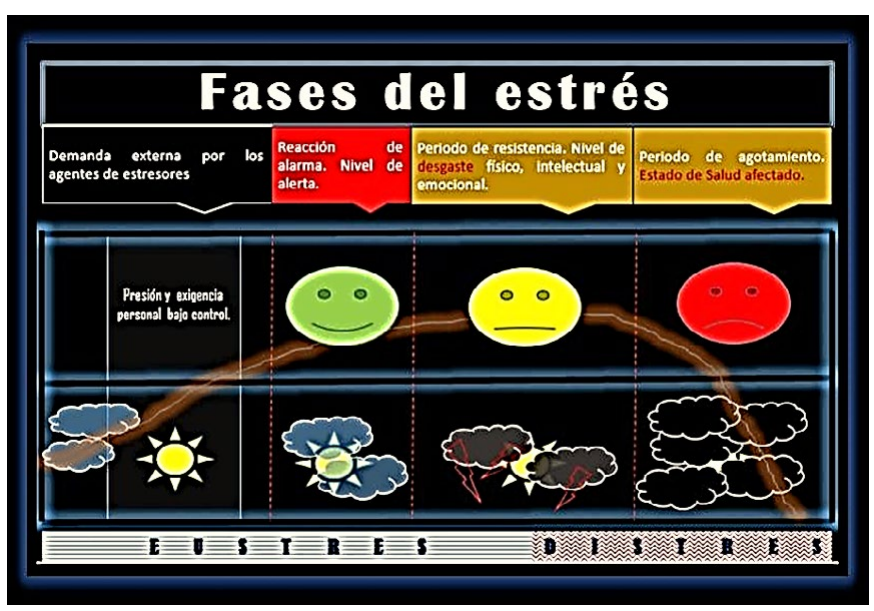

Figure 1. Stress phases.

Freudenberger [5-9], who chose the same word Burnout ("burnt", "consumed", "off") that was also used to refer to the effects of chronic use of toxic substances of abuse. This was a word commonly used in athletic, sports and artistic jargon, referring to those subjects who were not getting the expected results despite the efforts made.

Burnout syndrome, have symptoms ranging from deterioration and progressive exhaustion coupled with a drastic reduction of energy, also often accompanied by a loss of motivation over time affects attitudes, manners and overall behavior.

\section{The subject}

Burnout suffering literally burns unable to counter with their level of response, the enormous stress factors from the work environment. "Coping is understood here as cognitive and behavioral efforts that are developed to manage stress and reduces tensions caused by both internal and external aversive situations that people face in the course of daily life (Lazarus) [10-12]."Coping has two main functions: to manipulate or alter the problem with causing disturbance environment (coping addressed the problem), or regulate the emotional response that appears as a result (aimed at coping emotion). Both confrontations influence each other and can strengthen or interfere with each other."

\section{Conclusions}

As has-been broken down in this Article, the current society and its work demand caused by all previously named as phenomena: the globalization of the economy, advance communication through science, which shows every time Moreover, the growing process of hiring workers outside the borders by outsourcing corporations. Increasingly, greater demands on the contemporary worker, not only in terms of management skills and tools in their profession, but also those who demand emotional intelligence, such as teamwork, public speaking, leadership, etc., in addition to the strong competition among highly qualified personnel, who look postgraduate courses and have profoundly transformed the perception of stress at work $[13,14]$.

Therefore, a process of self-continuous monitoring is necessary to avoid stress, as part of the policy to be self-responsible through their perceptions. Their quality of life will depend on how capable it yourself, maintaining a margin of balance in the economy of time, ie, administering and above all, dosing it at work, recreation and rest. Knowing creator released their own decisions and participates.
It is imperative to always have those valves, such as traveling and spending time with family, friends and like-minded people who fill the wishes of affiliation and stress, and lead a healthy life, with a status of moderate planning, balancing exercise routines, nutritious meals based on healthy diets, quantity and quality of time devoted to sleep, rest and pleasurable recreational activities and above all, the fact of being at peace with oneself, maintaining a healthy mental and emotional activity, that is watching to transform negative thoughts into productive, positive and optimistic thoughts.

No need to burn at work, to be highly efficient, if we still have some ability to develop, will always be in our hands, to plan a reasonable time to enable us work those weaknesses. This insurance, which therefore have a positive effect on our level of productivity and efficiency at work.

\section{Conflict of Interest}

The views presented in this article represent only the author's view on the issues discussed.

\section{References}

1. McLuhan M (1973) The Guttenberg Galaxy, Herder: Barcelona, Issue 62.

2. Darwin ch (1880) Origin of Species by Means of Natural Selection or the Preservation of races in their struggle for existence. Godinez, e. (translation) (2nd edition, revised and enlarged edition). Madrid: Lucuix and Company.

3. Frankl V (1991) Man completo Search for Meaning, Herder: Barcelona, Issue 12.

4. Selye H (1974) Stress without distress.

5. Freudenberger H (1998) Staff burnout. Journal of Social Issues: 5-16.

6. Psychology of acta Colombiana (2007) Burnout Syndrome burned staying in the job burnout, 10: 117-125.

7. Bunge M (1960) Science, its method and its philosophy, Buenos Aires: Twentieth Century.

8. Bunge M (1961) Causality. The principle of causality in modern science, Buenos Aires: Eudeba.

9. Hernandez GL, Olmedo E (2004) Ibanez burnout (burnout) and its relationship with coping. International Journal of Clinical and Health Psychology 4: 323-336.

10. Luttmann to Jager M, Griefahn B (2004) Preventing musculoskeletal disorders, workplace. Switzerland: WHO.

11. Maslach C, Jackson S (1981) The measurement of experienced burnout. Journal of Occupational Behavior 2: 99-113.

12. Maslach C (1981) Measurement of experience burnout. Journal of Organizational Behavior.

13. Ornstein r (1979) Psychology of consciousness, Mexico: modern Manual.

14. Perez J (2004) Comparative analysis of the legislation on motor disorders caused by cumulative trauma as occupational disease, Mexico: University of Guanajuato, Ergonomists Society of Mexico, AC.

Copyright: (C2016 Medina JAP. This is an open-access article distributed under the terms of the Creative Commons Attribution License, which permits unrestricted use, distribution, and reproduction in any medium, provided the original author and source are credited. 